\title{
Injectable hydrogel composite containing modified gold nanoparticles: implication in bone tissue regeneration
}

This article was published in the following Dove Press journal: International Journal of Nanomedicine

\author{
Donghyun Lee' \\ Dong Nyoung $\mathrm{Heo}^{2}$ \\ $\mathrm{Ha}$ Ram $\mathrm{Nah}^{3}$ \\ Sang Jin Lee ${ }^{1}$ \\ Wan-Kyu Ko ${ }^{4}$ \\ Jae Seo Lee ${ }^{3}$ \\ Ho-Jin Moon' \\ Jae Beum Bang ${ }^{5}$ \\ Yu-Shik Hwang ${ }^{6}$ \\ Rui L Reis ${ }^{1,7}$ \\ II Keun Kwon'
}

'Department of Dental Materials, School of Dentistry, Kyung Hee University, Seoul 02447, Republic of Korea; ${ }^{2}$ Department of Engineering Science and Mechanics, Pennsylvania State University, Pennsylvania 16802, USA; ${ }^{3}$ Department of Detistry, Graduate School, Kyung Hee University, Seoul 02447, Republic of Korea; ${ }^{4}$ Department of Neurosurgery, CHA Bundang Medical Center, CHA University, Seongnam-si, Gyeonggi-do 13496 , Republic of Korea; ${ }^{5}$ Department of Dental Education, School of Dentistry, Kyung Hee University, Seoul 02447, Republic of Korea; ${ }^{6}$ Department of Maxillofacial Biomedical Engineering, School of Dentistry, Kyung Hee University, Seoul 02447, Republic of Korea; ${ }^{7}$ The Discoveries Centre for Regenerative and Precision Medicine, Headquarters at University of Minho, Barco, Guimarães, Portugal

Correspondence: II Keun Kwon Department of Dental Materials, School of Dentistry, Kyung Hee University, 26, Kyungheedae-ro, Dongdaemun-gu, Seoul 02447, Republic of Korea

$\mathrm{Tel}+8229610350$

Fax +82296I 9594

Email kwoni@khu.ac.kr
Background: For effective bone regeneration, it is necessary to implant a biocompatible scaffold that is capable of inducing cell growth and continuous osteogenic stimulation at the defected site. Here, we suggest an injectable hydrogel system using enzymatic cross-linkable gelatin (Gel) and functionalized gold nanoparticles (GNPs).

Methods: In this work, tyramine (Ty) was synthesized on the gelatin backbone (Gel-Ty) to enable a phenol crosslinking reaction with horseradish peroxidase (HRP). N-acetyl cysteine (NAC) was attached to the GNPs surface (G-NAC) for promoting osteo-differentiation.

Results: The Gel-Ty hydrogels containing G-NAC (Gel-Ty/G-NAC) had suitable mechanical strength and biocompatibility to embed and support the growth of human adipose derived stem cells (hASCs) during a proliferation test for three days. In addition, G-NAC promoted osteodifferentiation both when it was included in Gel-Ty and when it was used directly in hASCs. The osteogenic effects were demonstrated by the alkaline phosphatase (ALP) activity test.

Conclusion: These findings indicate that the phenol crosslinking reaction is suitable for injectable hydrogels for tissue regeneration and G-NAC stimulate bone regeneration. Based on our results, we suggest that Gel-Ty/G-NAC hydrogels can serve both as a biodegradable graft material for bone defect treatment and as a good template for tissue engineering applications such as drug delivery, cell delivery, and various tissue regeneration uses.

Keywords: nanomaterial, gelatin, enzymatic cross-linking, osteogenesis, $N$-acetyl cysteine

\section{Introduction}

Bone defects are caused by loss of a part of the bone due to trauma, osteomyelitis, or bone tumors. In order to treat bone defects, autologous, allogenic, and synthetic bone implants have been used in the clinical area. Nearly 2 million bone implants have been performed in the USA over the past 16 years. ${ }^{1}$ Recently, there has been an increase in artificial bone grafting because autologous bone grafting requires additional surgery for collecting bone tissue at other sites to be used for implantation. ${ }^{1-4}$ However, there are some disadvantages to artificial bone such as possible immune response, early absorption, and a lack of osteogenic factors..$^{5}$ To improve these problems, many studies have been performed on growth factors, drugs, and proteins that induce effective bone regeneration. ${ }^{6-9}$

In general, nanomaterials have a size of less than $100 \mathrm{~nm}$ and are manufactured in various sizes, shapes, and crystal structures using materials such as silicon, carbon, silver, and graphene. Nanomaterials have attracted much attention in the fields of chemistry, physics, and biotechnology due to their ease of mass production and wide range of applications. ${ }^{10,11}$ Among these materials, gold nanoparticles (GNPs) have better biocompatibility and surface specificity than other nanomaterials. For these reasons, 
GNPs have been widely applied in the fields of drug delivery, diagnosis, and tissue regeneration. ${ }^{12-15}$ In particular, GNPs are suitable for use as functional materials for bone regeneration because of their excellent effects of promoting bone differentiation of stem cells and osteoclast inhibition. ${ }^{12,16-19}$ In this study, $N$-acetyl cysteine (NAC) was grafted to GNPs by the surface specificity of GNPs for inducing a synergy effect for promoting bone regeneration.

NAC has been used clinically for kidney disease, hemorrhagic cystitis, obstructive pulmonary disease, and especially as a health supplement due to its antioxidant activity. ${ }^{20,21}$ Yamada et al have demonstrated that NAC enhances bone regeneration at a certain concentration by promoting osteogenesis-related gene expression such as osteopontin and osteocalcin. ${ }^{22}$ In addition, adipogenic differentiation was inhibited by intracellular antioxidant activity through NAC. ${ }^{23}$ NAC can be immobilized on GNP surfaces through covalent bonds by a reaction with the thiol group of NAC. After the NAC-grafted GNPs (G-NAC) were absorbed into the cell, the NAC separated by intracellular glutathione. ${ }^{13}$ Based on these theories, it is suggested that G-NAC was absorbed well into the cells by GNPs, and then GNPs and NAC will both express their activity in the cells.

Hydrogels are a type of material that imitate extracellular matrix in the body as they contain a lot of water due to the cross-linking of the hydrophilic polymer. Hydrogels contain water similar to the body environment and thus can provide an optimal environment for preserving the structure and physiological activity of hydrophilic proteins. ${ }^{24}$ In addition, it is possible to control the release of drugs, proteins, growth factors, and other substances contained within hydrogels. ${ }^{25,26}$ An injectable hydrogel, which induces a cross-linking reaction after implantation in the defects, can be brought into close contact with the surrounding structure regardless of the complexity of the shape of the defect. ${ }^{27,28}$ Biodegradable protein-based hydrogels, such as collagen, gelatin, silk fibroin, and chitosan, have been continuously studied for complete autologous tissue regeneration. Among these, gelatin is a material obtained from collagen, which is a fibrous protein that constitutes a substantial part of living tissue. ${ }^{24}$ It has a lower molecular weight than collagen, excellent biodegradability, biocompatibility, and high commercial potential. Also, it has favorable properties for bioapplication because it contains factors in collagen, such as the RGD sequence, which positively affects cell adhesion. ${ }^{29}$ However, formulation methods are still being researched as it is difficult to impart specific functions to the surface of gelatin such as what is done with other materials.

In this study, we designed G-NAC, a functional nanoparticle to promote bone formation, which was characterized chemically and physically. Subsequently, it was evaluated for cytotoxicity and promotion of bone differentiation in human adipose-derived stem cells (hASCs). The gelatin hydrogel was generated to induce tissue regeneration and allow G-NAC to continue to function (Figure 1). Gelatin with phenolic hydroxyl group was synthesized (Gel-Ty) by binding gelatin and tyramine (Ty) for hydrogel formation. Gel-Ty formed into a hydrogel by a reaction with horseradish peroxidase (HRP) and hydrogen peroxide $\left(\mathrm{H}_{2} \mathrm{O}_{2}\right)$. Biocompatibility was assessed both with and without the Gel-Ty hydrogel

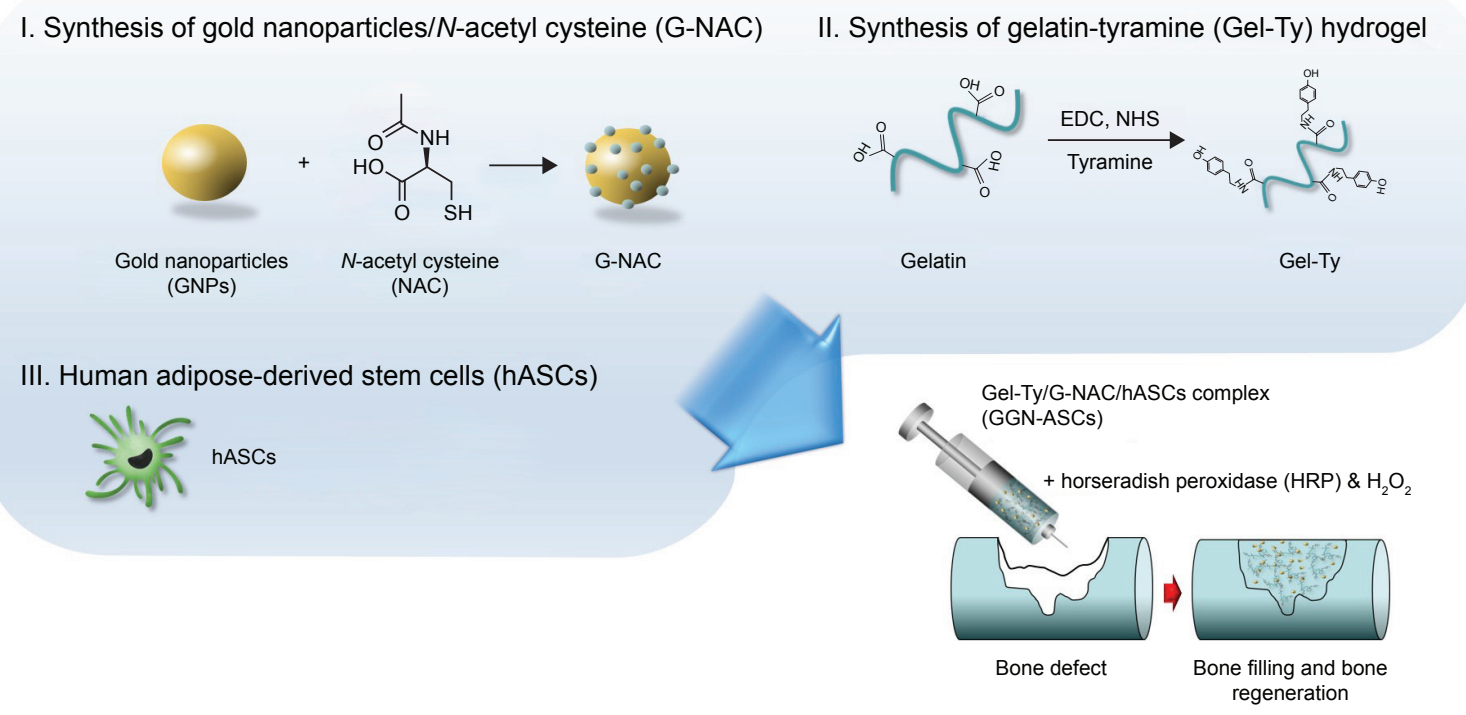

Figure I Schematic diagram of the GGN-ASCs injectable hydrogel production.

Abbreviation: GGN-ASCs, gelatin-tyramine/gold-nanoparticles- $N$-acetyl cysteine/human adipose-derived stem cells complex hydrogel; $\mathrm{H}_{2} \mathrm{O}_{2}$, hydrogen peroxide; EDC, I-ethyl-3-(3-dimethylaminopropyl)-carbodiimide hydrochloride; NHS, N-Hydroxysuccinimide. 
via hASCs. In addition, GNPs were encapsulated in Gel-Ty hydrogel with hASCs to assess bioavailability.

\section{Materials and methods Materials}

Gelatin type B, 2-morpholinoethanesulfonic acid (MES), sodium chloride, gold (III) chloride hydrate, sodium citrate, NAC and Ty were purchased from Sigma-Aldrich (St Louis, MO, USA). N-Hydroxysuccinimide (NHS) and 1-ethyl-3-(3dimethylaminopropyl)-carbodiimide hydrochloride (EDC) were purchased from Tokyo Chemical Industry (Tokyo, Japan). Ty, HRP, and Alexa Fluor 488 Phalloidin were purchased from Invitrogen (Thermo Fisher Scientific, Waltham, MA, USA). Spectra/Por 4 dialysis tubing (3.5 and $10 \mathrm{kDa}$ MWCO) was purchased from Spectrum Laboratories Inc (Rancho Dominguez, CA, USA). DMEM, FBS, antibiotic agents (penicillin/streptomycin, PS), TrypLE ${ }^{\mathrm{TM}}$ Express, PBS, and Dubecco's phosphate buffered saline (DPBS) were purchased from Gibco (Thermo Fisher Scientific). Deionizeddistilled water (DW) was produced by an ultrapure water system (Puris-Ro800; Bio Lab Tech., Republic of Korea).

\section{Equipment}

Proton nuclear magnetic resonance ( ${ }^{1} \mathrm{H}$ NMR) spectra were recorded on a Bruker Avance 400 (Bruker Corporation, Billerica, MA, USA). The viscoelastic properties were measured by a rotating rheometer (AR-G2, TA Instruments, New Castle, DE, USA). The fluorescently labeled cells were observed by a confocal laser scanning microscopy (CLSM, Eclipse E600W, Nikon Corporation, Tokyo, Japan). ELISAs were performed using a Benchmark Plus microplate spectrophotometer system (Bio-Rad Laboratories Inc, Hercules, CA, USA). Dynamic light scattering (DLS) was performed using an ELSZ-1000 (Photal, Otsuka Electronics, Osaka, Japan). Thermogravimetric analysis (TGA) was performed using an SDT Q600 (TA Instruments). Cell uptake of nanoparticles analysis was observed using a DM2500 microscope with dark-field (DF) filter (Leica Microsystems, Wetzlar, Germany).

\section{Synthesis and preparation of Gel-Ty/ G-NAC composite}

Synthesis and preparation of GNPs and G-NAC

The GNPs were produced using the citrate reduction of $\mathrm{HAuCl}_{4}$ as previously described..$^{30}$ Briefly, a $2 \%$ sodium citrate solution was rapidly added in a $0.02 \% \mathrm{HAuCl}_{4}$ solution at $100^{\circ} \mathrm{C}$. The reaction temperature was maintained for 15 minutes after the mixture turned to dark-purple and then bench cooled to room temperature. The GNP solution was filtrated using a $22 \mu \mathrm{m}$ syringe filter (EMD Millipore, Billerica, MA, USA). For attaching NAC on the GNPs surface, $580 \mu \mathrm{M}$ GNP solution was reacted with $29 \mathrm{mM}$ NAC solution and stirred for 48 hours at room temperature. The mixture was dialyzed for 5 days using a 3,500 Da dialysis membrane to remove unconjugated NAC.

\section{Synthesis and preparation of Gel-Ty hydrogels}

The MES buffer was prepared by blending $50 \mathrm{mM}$ MES and $0.5 \mathrm{M}$ sodium chloride in DW and then adjusting the $\mathrm{pH}$ to 6.0. Gelatin type B was dissolved in $60^{\circ} \mathrm{C}$ MES buffer at $2 \%$ concentration. After cooling, $25 \mathrm{mM}$ NHS and $50 \mathrm{mM}$ EDC were added at room temperature. After 1-hour reaction, Ty was added in half the amount of gelatin type B and was stirred for 24 hours at room temperature. The mixture was dialyzed for 7 days against DW using a $10 \mathrm{kDa}$ dialysis tube and then the dialyzed solution was lyophilized. The synthesized Gel-Ty was analyzed by ${ }^{1} \mathrm{H}-\mathrm{NMR}$. The Gel-Ty hydrogels were formed by reaction with HRP and $\mathrm{H}_{2} \mathrm{O}_{2}$. Briefly, 5 units of HRP and $25 \mu \mathrm{L}$ of $50 \mathrm{mM}$ $\mathrm{H}_{2} \mathrm{O}_{2}$ were mixed in PBS in which lyophilized Gel-Ty was dissolved at the required concentrations. The Gel-Ty solution reacted into the form of a hydrogel within 1 minute. The hydrogels were prepared as a film with a thickness of $1 \mathrm{~mm}$ and shaped into an $8 \mathrm{~mm}$ diameter disk using a biopsy punch. These disks were used for rheometer and cell experiments. The viscoelastic properties of Gel-Ty hydrogels were measured using a rotating rheometer. For rheological analysis, the samples were compressed to $5 \%$ strain at frequencies from 0.1 to $100 \mathrm{~Hz}$. The temperature was controlled at $25^{\circ} \mathrm{C}$ during the viscoelastic measurement.

\section{Preparation of Gel-Ty/GNPs composite hydrogels}

Solutions of 20, 100, and $200 \mu \mathrm{M}$ GNPs and G-NAC solutions were centrifuged at 13,000 rpm for 15 minutes to settle the particles. The supernatant-removed pellets were mixed in $7.5 \%$ Gel-Ty solution and then they were reacted with HRP, $\mathrm{H}_{2} \mathrm{O}_{2}$, and hASCs. The Gel-Ty hydrogels formed contained 10,100, 200, and $400 \mu \mathrm{M}$ GNPs, respectively. These were used for in vitro experiments.

\section{Characterizations of G-NAC}

The synthesized GNPs and G-NAC were characterized by DLS analysis to confirm the size of the nanoparticles at a concentration of $200 \mu \mathrm{M}$ at $25^{\circ} \mathrm{C}$. Before ultraviolet-visible (UV-vis) spectrum analysis, the concentration of the GNP solution was concentrated because the concentration of 
G-NAC solution was decreased when it was dialyzed during the production process. GNPs and G-NAC were centrifuged at 13,000 rpm for 15 minutes and then were lyophilized for TGA. TGA was performed under a high purity nitrogen flow of $100 \mathrm{~mL} / \mathrm{min}$. The temperature of the furnace was set to increase at $10^{\circ} \mathrm{C} / \mathrm{min}$ and up to a maximum of $800^{\circ} \mathrm{C}$.

\section{Human adipose-derived stem cells (hASCs) culture}

The hASCs (Cefobio, Seoul, Republic of Korea) were cultured in growth medium (GM) or osteodifferentiation medium (OM) at $37^{\circ} \mathrm{C}$ in $5 \% \mathrm{CO}_{2}$ incubator (Isotemp, Thermo Fisher Scientific). The GM was composed of DMEM, 10\% FBS, and 1\% PS. The OM was composed of GM, $10 \mathrm{mM} \beta$-glycerol phosphate disodium salt hydrate, $300 \mu \mathrm{M}$ ascorbic acid, and $0.1 \mu \mathrm{M}$ dexamethasone. In the all cell experiments using hASCs, and the GM and OM were changed every 3 days.

\section{Intercellular uptake analysis of GNPs and G-NAC}

The intercellular uptake of GNPs and G-NAC was determined by DF assay. ${ }^{12}$ The hASCs were seeded in a confocal dish (SPL, Pocheon, Republic of Korea) and treated with $20 \mu \mathrm{M}$ GNPs and G-NAC solutions. After 12 hours, these were fixed using 3.7\% formaldehyde solution and were observed through a DM2500 microscope equipped with a DF filter. DF images were analyzed by ImageJ program.

\section{Viability of hASCs analysis}

The viability of hASCs was evaluated using fluorescence staining and cell viability assay kit (EZ-Cytox, Dogne, Republic of Korea). For the evaluation of GNPs, NAC, and G-NAC, hASCs were seeded at a density of $1 \times 10^{4}$ cells per well in GM. After 1 day, GM was changed to various GM containing various concentrations of GNPs, NAC, and G-NAC, respectively. These were then reacted by $1 / 10$ diluted EZ-Cytox solution for 90 minutes, and the reacted solutions were measured using an ELISA reader operating at $450 \mathrm{~nm}$ wavelength. In the evaluation of Gel-Ty hydrogels, two methods were used to confirm the surface and internal properties of hydrogels. First, for the evaluation on the hydrogel surface, a hydrogel prepared in the form of $8 \mathrm{~mm}$ diameter disk was placed on a 48 -well cell culture plate, and drop seeding was carried out at a concentration of $1 \times 10^{4}$ cells per sample. Second, for hASCs-embedded hydrogel preparation, two types of Gel-Ty solutions were prepared before the hydrogel cross-linking. The cross-linker
$\mathrm{H}_{2} \mathrm{O}_{2}$ was added to $10 \%$ Gel-Ty solution, and $2 \times 10^{6}$ cells $/ \mathrm{mL}$ hASCs were dispersed in the other $10 \%$ Gel-Ty solution with HRP. These two solutions were then mixed and poured using a dual syringe. The cross-linked hydrogels were cut to disks and then they were moved to 48-well cell culture plate. All hydrogel samples were evaluated using a cell viability assay kit. In addition, the hASC cultured hydrogels were fixed using $3.7 \%$ formaldehyde solution and then were stained using Alexa Fluor 488 phalloidin and DAPI. The prepared samples were observed by CLSM.

\section{ALP activity}

The hASCs were seeded in a 48 -well cell culture plate at a density of $2 \times 10^{4}$ cells per well or sample. After 1 day, GM was changed to either OM or OM containing GNPs, NAC, or G-NAC, respectively. The cells were then cultured for 5, 10, and 15 days. The osteodifferentiated hASCs were washed with DPBS and lysed with $3 \times$ RIPA buffer at $4^{\circ} \mathrm{C}$ for 1 hour. The lysed hASCs were centrifuged at 10,000 rpm for 10 minutes, and the supernatant was reacted with $p$-nitrophenol phosphate (Sigma-Aldrich) in a $37^{\circ} \mathrm{C}$ incubator for 30 minutes. The level of $p$-nitrophenol production was measured using an ELISA reader at $405 \mathrm{~nm}$ wavelength.

\section{Statistical analysis}

For hASC viability and ALP activity tests, a known quantity of hASCs was seeded in four wells per group. In addition, statistical analysis was performed using two-way ANOVA with Tukey's multiple comparison post hoc tests. All values are expressed as the mean $\pm \mathrm{SD}$, and all the experimental groups are compared with each other. Significance was defined as $* P<0.05$, ** $P<0.01$, and $* * * P<0.001$.

\section{Results}

\section{Characterization of GNPs and G-NAC}

DLS analysis of GNPs at $580 \mu \mathrm{M}$ concentration showed that the particles had diameters mostly in the range of $28-32 \mathrm{~nm}$. The average diameter of particles was $35.83 \pm 0.9 \mathrm{~nm}$, and polydispersity (PDI) was measured as $0.3000 \pm 0.026$ (Figure 2A). The GNP solution was prepared with G-NAC through reaction with NAC solution. The GNPs and G-NAC were measured using UV spectrophotometer. The measured maximum absorbance values were 522.5 and $527 \mathrm{~nm}$ for GNPs and G-NAC, respectively. Here, the graph of G-NAC is similar to the graph of GNPs, but it is confirmed that it has moved to the right of the graph of GNPs. The TGA graph showed the pyrolytic properties of GNPs, G-NAC, and NAC when these were heated to $800^{\circ} \mathrm{C}$ (Figure $2 \mathrm{C}$ ). The mass 

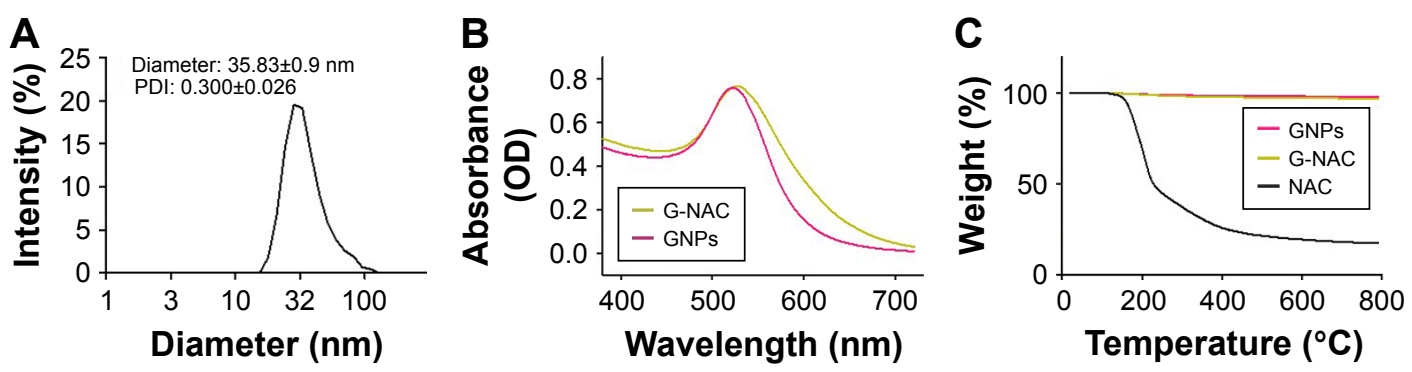

Figure 2 Identification and quantification of GNPs and G-NAC.

Notes: DLS spectrum of GNPs (A), UV-vis spectrum of GNPs and G-NAC (B), and TGA curves of GNPs, NAC, and G-NAC (C). The weight is constant up to $800^{\circ} \mathrm{C}$. Abbreviations: DLS, dynamic light scattering; G-NAC, gold nanoparticles-N-acetyl cysteine; GNPs, gold nanoparticles; NAC, N-acetyl cysteine; TGA, thermogravimetric analysis; UV-vis, ultraviolet visible.

reduction rates of GNPs, G-NAC, and NAC were $2.177 \%$, $3.088 \%$, and $82.58 \%$, respectively.

\section{Viability and osteodifferentiation of hASCs by NAC, GNPs, and G-NAC}

We confirmed how NAC and GNPs, independently, affect hASCs (Figure 3). In Figure $3 \mathrm{~A}$ and $\mathrm{C}$ graphs, the group with no treatment of NAC or GNPs was set to control (100\%), and the remaining groups were compared with the control group. NAC was evaluated in the range of $0.01-50 \mathrm{mM}$. The concentration of $50 \mathrm{mM} \mathrm{NAC}$ was significantly lower $(79.9 \%$, $49.4 \%$, and $37.8 \%$ ) over all periods. All survival rates were similar or slightly increased in all groups, except for $50 \mathrm{mM}$ NAC. However, as the duration of drug treatment increased, the cell proliferation rate decreased at the groups treated with high concentrations of NAC. At 7 days, all NAC-treated groups, except the $0.01 \mathrm{mMNAC}$ group (265.8\%), were $22 \%$ lower than the control group (255.1\%). On the contrary, all groups with added GNPs had a higher cell viability than the control group. In the group treated with $40 \mu \mathrm{M}$ GNPs for 7 days, the value of viability was 1.73 times higher than the control group.

ALP activity analysis was performed to confirm the bone differentiation effect of NAC and GNPs. It was performed within the noncytotoxic range, based on the viability analysis
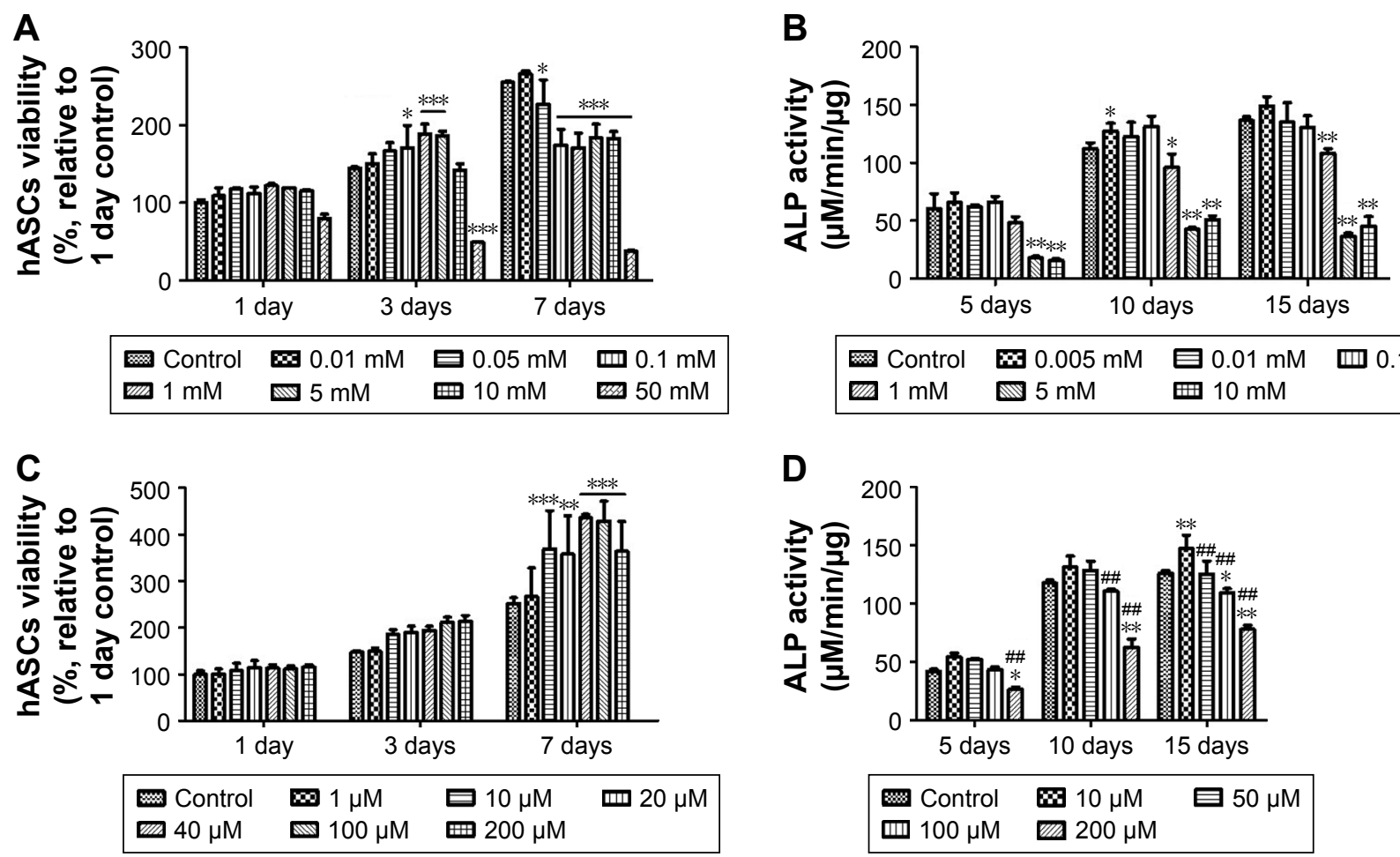

Figure 3 Evaluation of viability (A, C) and ALP activity (B, D) of hASCs at various concentrations of NAC (A, B) and GNPs (C, D). Results are mean \pm SD of triplicate experiments: $* P<0.05$, $* * P<0.01$, and $* * * P<0.00$ I represent significant difference compared with control group, and ${ }^{*} P<0.0$ I represent significant difference compared with I0 $\mu M$ group. Abbreviations: GNPs, gold nanoparticles; hASCs, human-derived stem cells; NAC, N-acetyl cysteine; ALP, Alkaline phosphatase. 
results of hASCs. The ALP activity of $1 \mathrm{mM}$ NAC was measured to be $48.4,96.1$, and $108.4 \mu \mathrm{M} / \mathrm{min} / \mu \mathrm{g}$ at 5,10 , and 15 days, respectively. This was significantly lower than the control group (Figure 3B). In the ALP activity of GNPs, $10 \mu \mathrm{M}$ GNPs was higher at all time periods. However, at concentrations above $50 \mu \mathrm{M}$, the ALP activity tended to decrease as the concentration of GNPs increased, unlike viability (Figure 3D). Thus, the concentration of GNPs was further subdivided into $50 \mu \mathrm{M}$ and the ALP activity was found to be the highest in the $20 \mu \mathrm{M}$ group (Figure S1).

Cell viability and ALP activity by G-NAC were assessed after treatment with the same concentrations as GNPs (Figure S2). During the viability of hASCs test, the values were significantly different from control (146.3\%) when 40,100 , and $200 \mu \mathrm{M}$ of G-NAC were treated for 3 days. At 7 days, the hASCs viability was 1.65 times higher than control in $100 \mu \mathrm{M}$ G-NAC group. The ALP activity by G-NAC was highest when treated with $10 \mu \mathrm{M}$, and the values were higher than the 50,100, and $200 \mu \mathrm{M}$ G-NAC groups.

Based on the above results, a comparative evaluation of GNPs, NAC, and G-NAC for viability and ALP activity was performed. GNPs and G-NAC were treated at a concentration of $20 \mu \mathrm{M}$ and NAC at a concentration of $2.2 \mu \mathrm{M}$ (Figure 4 ). The NAC-treated group at 7 days showed 0.85 -fold the control group for viability, but the values of ALP activity were 1.17-, 1.16-, and 1.09-fold the control group at 5, 10, and 15 days, respectively. GNPs and G-NAC showed higher cell viability than the control group, but there was no significant difference between them. However, ALP activity showed a significant difference, by a maximum of 1.18 times and a minimum of 1.08 times higher than GNPs in G-NAC.

\section{Intercellular uptake of GNPs and G-NAC}

The hASCs were cultured in confocal dishes and then treated with $20 \mu \mathrm{M}$ of GNPs and G-NAC, respectively, to observe the uptake of GNPs and G-NAC into the cells (Figure 5). The absorption of GNPs and G-NAC into the cells was observed in the bright field image (Figure 5A and $\mathrm{C}$ ). In the DF image, however, only the absorbed particles were observed in white and there is little difference in absorbed amount. DF images were quantitatively compared using the ImageJ program, resulting in almost no difference between GNPs (2.38\%) and G-NACs (2.27\%) (Figure 5E).

\section{Preparation and characterization of Gel-Ty hydrogel}

In the ${ }^{1} \mathrm{H}$ NMR analysis of synthesized Gel-Ty and gelatin, the unique peaks were detected in Gel-Ty at 7.2 and $6.9 \mathrm{ppm}$ (Figure 6A). The synthesized Gel-Ty was in a liquid state when it was dissolved in PBS, but retained a viscoelastic solid form after $\mathrm{HRP}$ and $\mathrm{H}_{2} \mathrm{O}_{2}$ were added (Figure 6C). The resulting Gel-Ty hydrogel was fixed at 5\% stress at room temperature using a rheometer, and the modulus was measured by increasing the frequency from 0.1 to $40 \mathrm{~Hz}$. The loss elastic modulus $\left(\mathrm{G}^{\prime \prime}\right)$ of the $5 \%, 6.5 \%$, and $8 \%$ Gel-Ty hydrogels was $4.32 \pm 0.86,5.32 \pm 0.96$, and $6.56 \pm 0.71$, respectively. However, the storage modulus $\left(\mathrm{G}^{\prime}\right)$ greatly increased in proportion to the increase of Gel-Ty concentration (Figure 6B).

\section{Viability and ALP activity of hASCs on the Gel-Ty and Gel-Ty/G-NAC hydrogels}

The hASC viability was evaluated in and out of the hydrogel to determine the optimum concentration of Gel-Ty for loading G-NAC. Cells cultured on 5\%-8\% Gel-Ty surface were fluorescently stained and confirmed that they proliferated well in all groups using CLSM (Figure S3A-F). In addition, there was no significant difference at all concentrations of Gel-Ty as shown in Figure S3G. However, at 7 days, the values of all hydrogel groups were 1.5 times higher than the hASCs cultured in the tissue culture plate
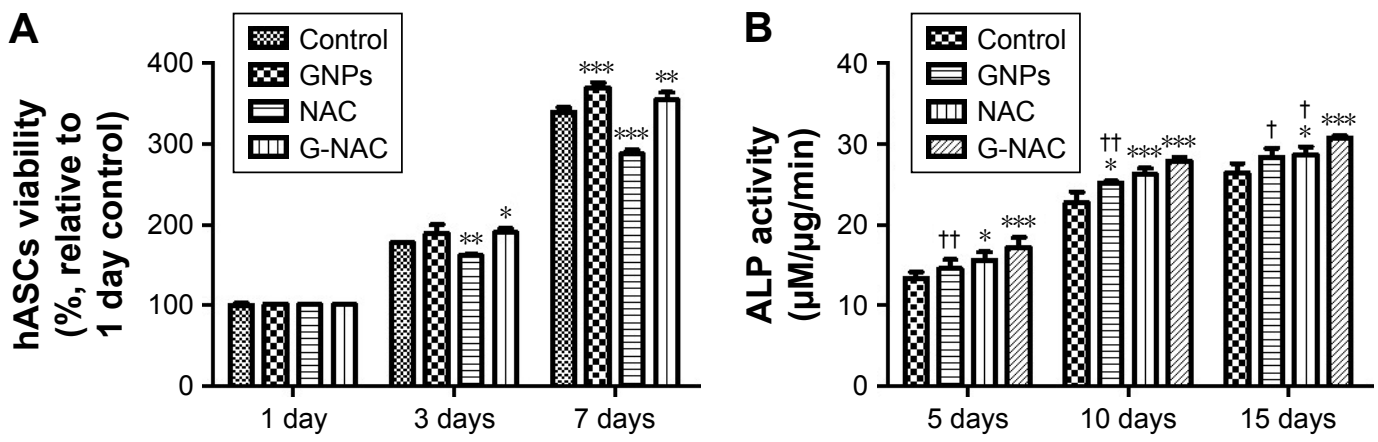

Figure 4 Evaluation of viability (A) and ALP activity (B) of hASCs at $20 \mu M$ GNPs, $2.2 \mu M$ NAC, and $20 \mu M$ G-NAC. Results are mean \pm SD of triplicate experiments: $* P<0.05$, $* * P<0.01$, and $* * * P<0.001$ represent significant differences compared with control group, and ${ }^{\dagger} P<0.05$ and ${ }^{\dagger+} P<0.01$ represent significant differences compared with G-NAC group.

Abbreviations: G-NAC, gold nanoparticles-N-acetyl cysteine; GNPs, gold nanoparticles; hASCs, human-derived stem cells; NAC, N-acetyl cysteine. 

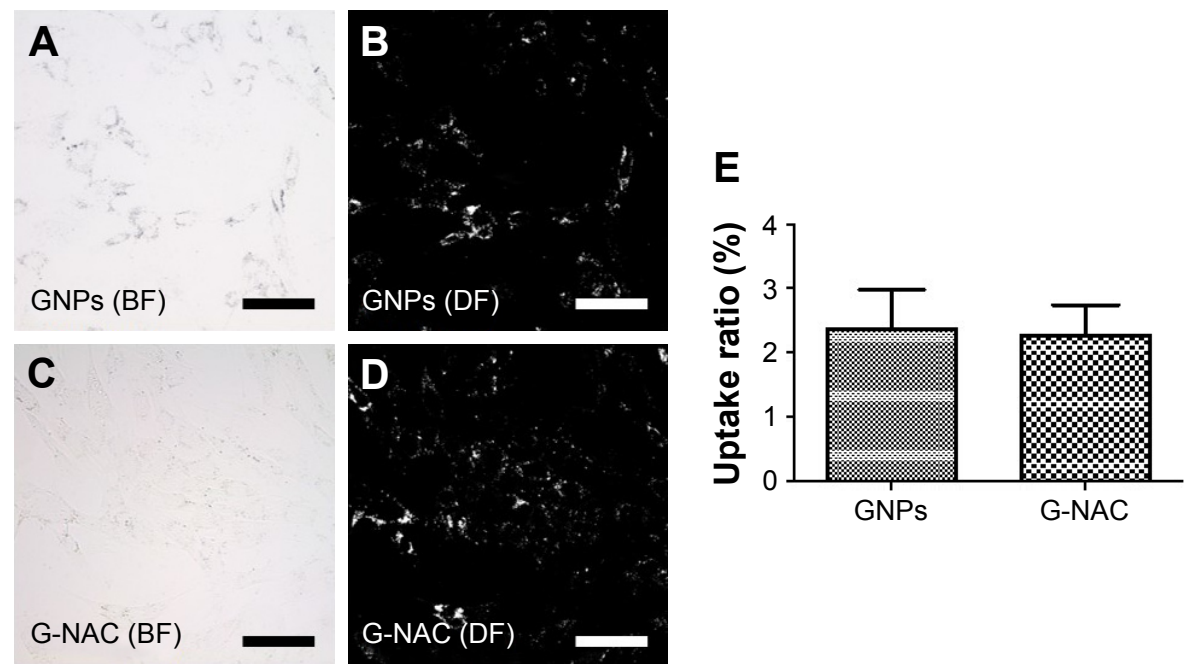

Figure 5 BF and DF images of hASCs treated with $20 \mu$ M GNPs (A, B) and G-NAC (C, D) for I day. The DF images evaluated by Image program (bright pixel/total pixel), and the results are mean \pm SD of triplicate experiments (E). Scale bars are $100 \mu \mathrm{m}$.

Abbreviations: BF, bright field; DF, dark field; G-NAC, gold nanoparticles-N-acetyl cysteine; GNPs, gold nanoparticles; hASCs, human-derived stem cells.

set as the control group. The number of living cells in the hydrogels was similar among the groups (Figure 7M), but the shape of $5 \%$ and $7.5 \%$ Gel-Ty hydrogels did not maintain well after 3 days compared with 10\% Gel-Ty groups (Figure 7A-L). To evaluate the applicability of GNPs to Gel-Ty hydrogels, cell viability was measured by preparing $10 \%$ Gel-Ty complexes with various concentrations of GNPs (Figure $7 \mathrm{~N}$ ). As a result, it was confirmed that the cell viability was increased as the concentration of GNPs increased. Particularly, at the concentration of $200 \mu \mathrm{M}$ or more, there was a significant difference compared with the control group.
The ALP activity occurred at the Gel-Ty hydrogels containing various concentrations of GNPs (Figure 8A). Samples containing $100 \mu \mathrm{M}$ of GNPs showed the highest values compared with the other groups at the 5,10 , and 15 days and at least 1.33 times and 1.62 times higher than the samples without GNPs; 20 and $200 \mu \mathrm{M}$ GNPs were all better than controls, but they were lower than the samples containing $100 \mu \mathrm{M}$ GNPs. Based on these results, a comparative experiment was performed on the osteodifferentiation effects of Gel-Ty/GNPs and Gel-Ty/G-NAC containing $100 \mu \mathrm{M}$ of nanoparticles (Figure 8B). Gel-Ty/G-NAC values were 1.85 times and 1.41 times higher than Gel-Ty and Gel-Ty/GNP, respectively.
A

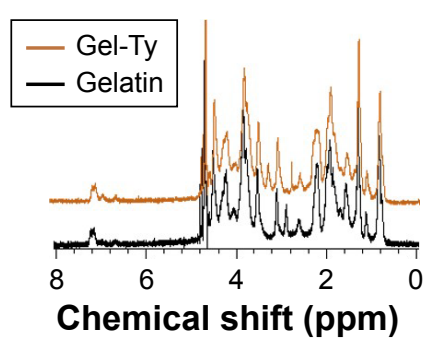

C

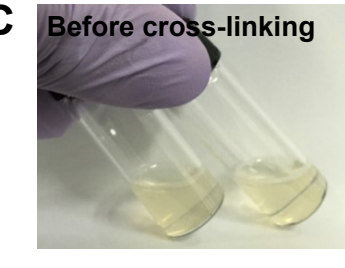

B
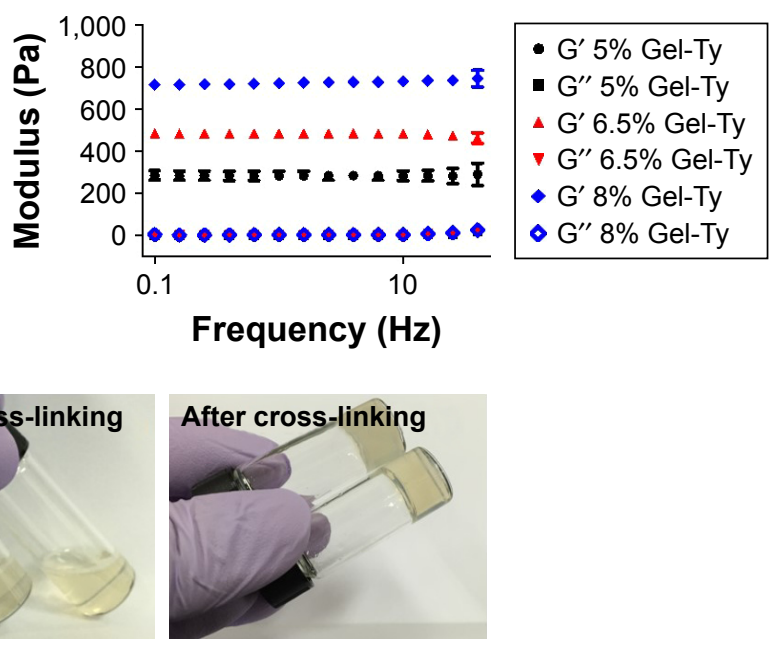

Figure 6 Characterization of Gel-Ty hydrogels.

Notes: 'H NMR spectrum of the gelatin and Gel-Ty (A). Image of $5 \%$ Gel-Ty solution, and cross-linked Gel-Ty hydrogel after addition of HRP and $\mathrm{H}_{2} \mathrm{O}_{2}$ (B). Frequency dependence of storage modulus $\left(G^{\prime}\right)$ and loss modulus $\left(G^{\prime \prime}\right)$ for various concentrations of Gel-Ty $(\mathbf{C})$. Results are means \pm SD of triplicate experiments. Abbreviations: Gel-Ty, gelatin-tyramine; 'H NMR, proton nuclear magnetic resonance; HRP, horseradish peroxidase; $\mathrm{H}_{2} \mathrm{O}_{2}$, hydrogen peroxide; Ppm, parts per million. 

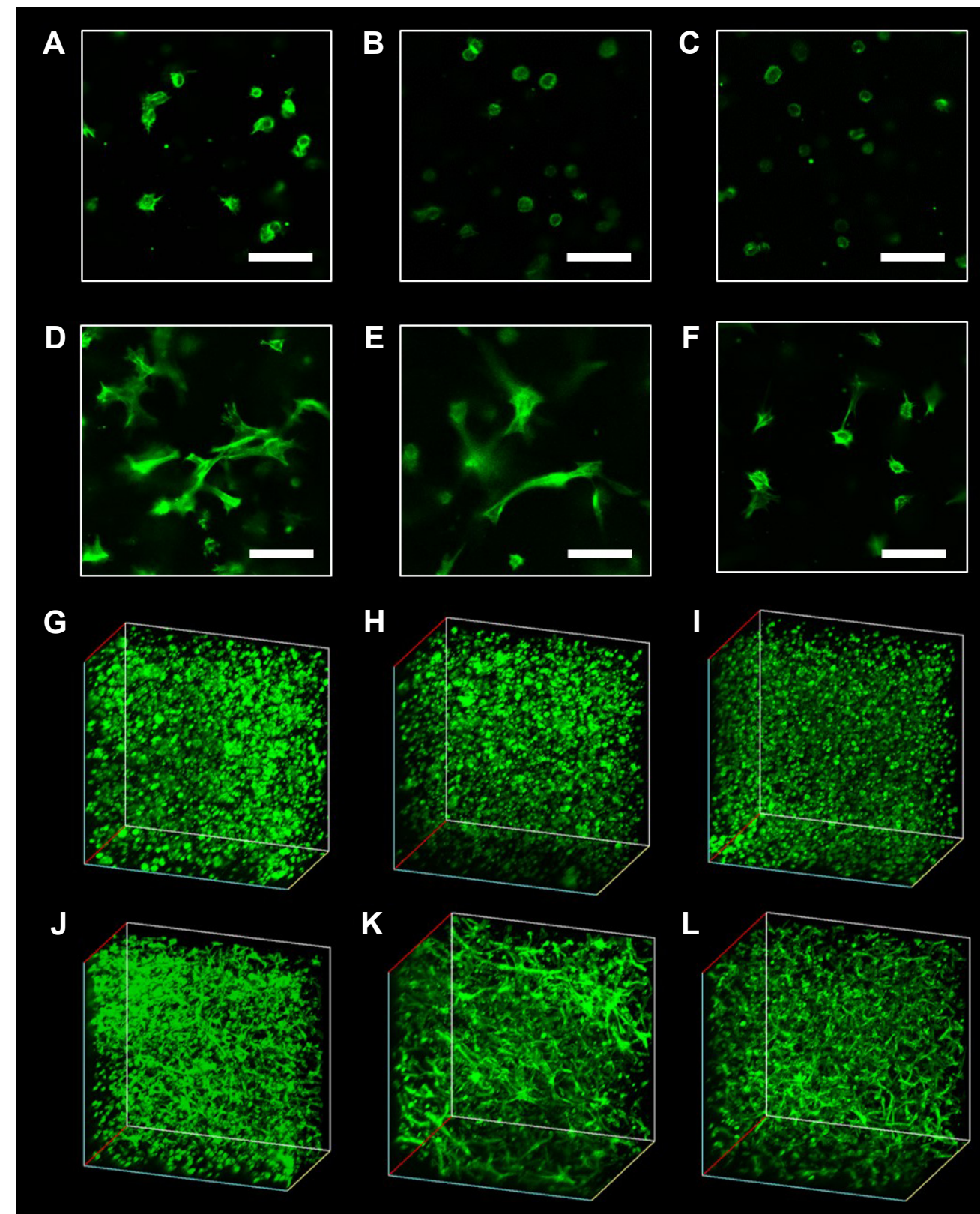

M

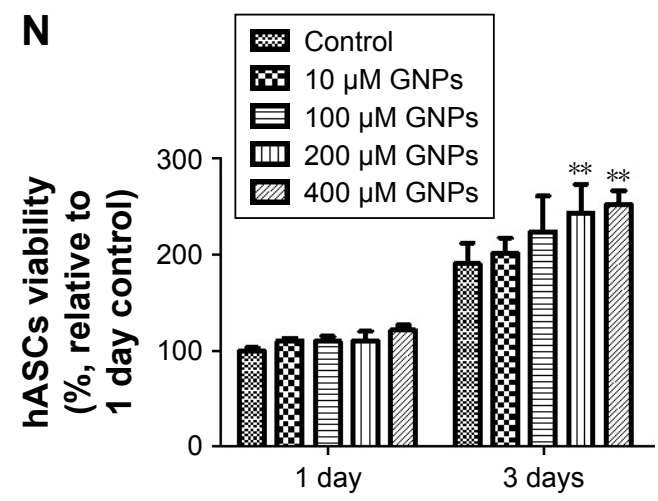

Figure 7 Viability of hASCs embedded in the Gel-Ty hydrogels.

Notes: The CLSM images of $5 \%$ (A, D, G, J), 7.5\% (B, E, H, K), and 10\% Gel-Ty hydrogel (C, F, I, L) after culture during I (A-C, G-I) and 3 days (D-F, J-L). F-actin of hASCs is green, and scale bar is $100 \mu \mathrm{m}$. The viability of hASCs embedded inside the Gel-Ty (M) and Gel-Ty/GNPs (N) hydrogels for 3 days. Results are mean \pm SD of triplicate experiments: $* * P<0.01$ represent significant differences compared with control group

Abbreviations: CLSM, confocal laser scanning microscopy; Gel-Ty, gelatin-tyramine; Gel-Ty/GNPs, gelatin-tyramine/gold nanoparticles; hASCs, human-derived stem cells. 

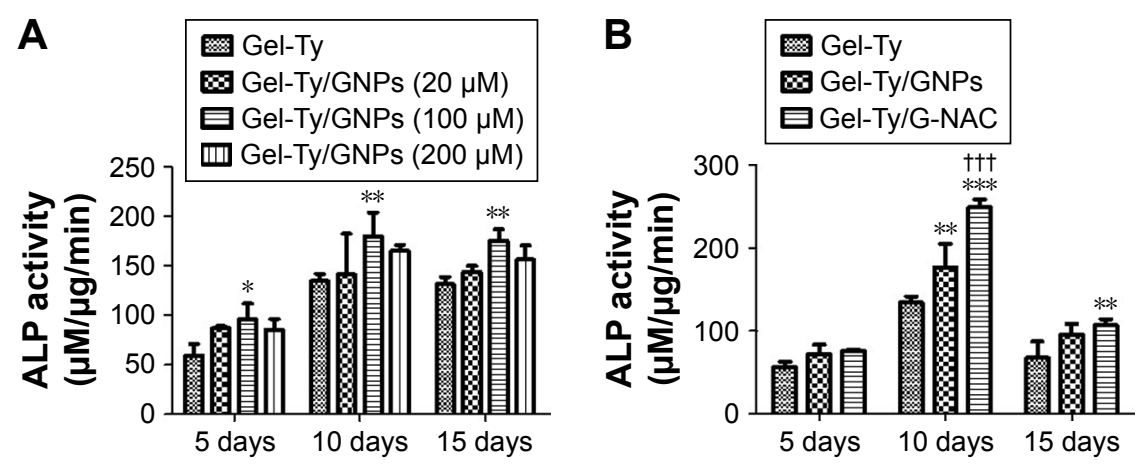

Figure 8 ALP activity of hASCs on the Gel-Ty/GNPs (A) and Gel-Ty/G-NAC (B).

Note: Results are means $\pm S D$ of triplicate experiments: $* P<0.05$ and $* * P<0.01$ represent significant difference compared with control group, $* * * P<0.00$ I represent significant difference compared with control group, and t+tP<0.00I represent significant difference compared with Gel-Ty/GNPs group.

Abbreviations: Gel-Ty/GNPs, gelatin-tyramine/gold nanoparticles; Gel-Ty/G-NAC, gelatin-tyramine/gold nanoparticles-N-acetyl cysteine; hASCs, human-derived stem cells.

\section{Discussion}

GNPs have been used in the biomedical field for a variety of applications such as drug delivery, diagnosis, anticancer, and tissue regeneration. ${ }^{13,31}$ For bone regeneration, GNPs are involved in the $\mathrm{p} 38$ mitogen-activated protein kinase signaling pathway when they are absorbed into mesenchymal stem cells (MSCs). This reaction induces osteoblast growth by activating osteoprogenitor factors and inhibiting adipogenic factors in the nucleus. ${ }^{17}$ Based on this theory, we have found positive results in several bone regeneration studies using GNPs. ${ }^{16,32}$ In the previous study, we reported that GNPs of 30 and $50 \mathrm{~nm}$ diameter were effective in increasing osteodifferentiation through the expression of the Runx2 gene as well as endocytosis. ${ }^{33}$ In addition, GNPs have different properties for organ accumulation and toxicity by diameter, with $30 \mathrm{~nm}$ diameter GNPs causing a decrease in leukocyte and red blood cells in the blood and accumulating in the spleen. This has been reported to be safer than other sizes. ${ }^{12,34}$ Therefore, we fabricated GNPs with a size of about $30 \mathrm{~nm}$ and confirmed that $35.83 \pm 0.9 \mathrm{~nm}$ GNPs were fabricated through DLS analysis. In the DLS results, the distribution range of 28-32 nm was the highest range and PDI was measured as 0.3 , which proved that GNPs of the desired size were uniformly produced (Figure 2A).

GNPs are produced by citrate reduction method, which is the most common method, and it is still widely used because it can be made simply by $\mathrm{DW}, \mathrm{HAuCl}_{4}$, and sodium citrate. ${ }^{30}$ The GNPs thus fabricated have surface area properties that easily couple with hydrophobic groups, anionic groups, and thiol groups. ${ }^{35} \mathrm{NAC}$, with a thiol group, easily bonds with GNPs. Due to the localized surface plasmon resonance characteristic of the gold surface, the absorption and scattering wavelengths are different depending on the organic matter when the organic material is bonded to the gold surface..$^{36,37}$
UV-vis spectrum was measured using the properties of these GNPs, and the maximum absorption wavelength of G-NAC was shifted $4.5 \mathrm{~nm}$ compared with GNPs (Figure 2B). As a result, the binding of NAC was demonstrated.

The NAC-synthesized G-NAC was quantitated by TGA. The difference in mass reduction between GNPs and G-NAC was $0.911 \%$ and NAC was $82.58 \%$ when the NAC, GNPs, and G-NACs were heated to $800^{\circ} \mathrm{C}$. The difference in mass reduction rates between G-NAC and GNPs is due to pyrolysis of the NAC bound to the GNPs surface. ${ }^{12}$ Thus, the mass difference between GNPs and G-NAC corresponded to $17.42 \%$ of the NAC mass. The mass of the combined NAC was calculated to be $5.23 \%$ of the GNPs mass. Zhu et al demonstrated that the intracellular uptake of functionalized GNPs was influenced by hydrophobicity and charge. ${ }^{38}$ Jiang et al reported that the rate of cell uptake depended on the size of the nanoparticles. ${ }^{39}$ Therefore, in this study, DF microscope assay was performed to determine whether NAC attachment affects cell uptake. In Figure 5, there was no significant difference in the cellular uptake of GNPs and G-NAC, suggesting that GNPs might be useful for intracellular delivery of NAC. G-NAC, which is absorbed into the cells, can be separated to GNPs and NAC by glutathione and, after cleavage, these act independently. ${ }^{13}$

The reactive oxygen species (ROS) refers to oxygen in a deformed and unstable state, such as superoxide ion and hydroxyl radical. Reduction of ROS in MSCs promotes osteogenesis by stimulating the Wnt and MAPK pathway while inhibiting adipogenesis through inhibiting CCAAT/ enhancer-binding proteins (CEBPs). ${ }^{23}$ We have applied NAC, a widely used antioxidant, to GNPs to accelerate bone differentiation. When NAC was used alone, the hASCs displayed cytotoxicity at concentrations exceeding $10 \mathrm{mM}$, so experiments were conducted at concentrations lower than that (Figure 3A). Figures 3 and 4 show that GNPs and 
G-NAC promote osteodifferentiation of hASCs especially at $20 \mu \mathrm{M}$ concentration. In addition, a small amount of NAC, as much as adding $20 \mu \mathrm{M}$ G-NAC, promoted osteodifferentiation similar to the $20 \mu \mathrm{M}$ GNPs, despite relatively low cell viability. A comparative evaluation of the ALP activity of GNPs, NAC, and G-NAC demonstrated that NAC bound to GNPs works to synergistically improve bone regeneration as shown by the highest ALP value in G-NAC (Figure 4).

We conjugated the amino group of Ty to the carboxyl groups of gelatin to bind the phenol group for the cross-linking reaction to the gelatin. As a result, gelatin with a hydroxyl phenol group was synthesized and the peaks of the phenyl protons of Ty present in Gel-Ty were observed at 7.2 and 6.9 ppm (Figure 6A). ${ }^{40}$ The synthesized Gel-Ty is dissolved in PBS. Subsequently, HRP and $\mathrm{H}_{2} \mathrm{O}_{2}$ are added to form a hydrogel through dehydrogenation between phenol analogs (Figure 6C). HRP, an alpha-helical protein bound to a heme group, acts as a catalyst for the radical generation of $\mathrm{H}_{2} \mathrm{O}_{2}$. One $\mathrm{H}_{2} \mathrm{O}_{2}$ reacts with HRP to produce two phenolic radicals, and these form hydrogels by causing cross-linking within the Gel-Ty by phenol groups. ${ }^{41}$ The Gel-Ty hydrogel crosslinking can be easily controlled depending on concentration and its stiffness was increased in proportion to concentration (Figure 6B). In addition, because the number of radicals generated according to the concentration of $\mathrm{H}_{2} \mathrm{O}_{2}$ or HRP varied, it can be used to control the mechanical strength or gelation time of the hydrogel. Thus, it has been used in many studies. ${ }^{42-47}$ Biocompatibility was assessed by incubating cells on the surface and inside of Gel-Ty hydrogel. Cell viability was not significantly different between hydrogels at all concentrations. However, as the hydrogel intensity decreased in the low concentration of Gel-Ty, it was degraded in a short time (Figure 7J, K and L). Therefore, 10\% Gel-Ty was selected as the hydrogel to apply G-NAC. The viability of hASCs inside the Gel-Ty hydrogel with various concentrations of GNPs was positively correlated with the number of GNPs, confirming the possibility of G-NAC loading (Figure 7N). Experiments in which GNPs are directly applied to hASCs were added to additional GNPs whenever media were replaced, but not when GNPs or G-NAC were contained in Gel-Ty hydrogels. Thus, hydrogel experiments with nanoparticles proceeded with higher concentrations of nanoparticles than the studies using only nanoparticles (Figure 8). The bone differentiation effect of Gel-Ty containing various concentrations of GNPs was highest at $100 \mu \mathrm{M}$ (Figure 8A). The GNPs and G-NAC were found to have the highest bone mineralization promoting effect at $20 \mu \mathrm{M}$, while the total amount of nanoparticles treated for 15 days was about
$100 \mu \mathrm{M}$ (Figures S1 and 4B). In addition, low ALP activity was measured at higher or lower concentrations of GNPs, similar to experiments in which only nanoparticles were used. As a result, it was once again confirmed that there is a proper concentration for maximizing the bone differentiation promoting effect. It was proved that G-NAC preserves its excellent bone differentiation promoting effects even when applied to Gle-Ty through the comparative study of Gel-Ty hydrogels containing $100 \mu \mathrm{M}$ GNPs and G-NAC.

\section{Conclusion}

In summary, we conducted an evaluation of GNPs and NAC, which are substances capable of promoting osteodifferentiation. Based on these results, we designed and prepared G-NAC, and the biocompatibility and osteodifferentiation promoting effects of G-NAC were demonstrated by the viability and ALP activity of hASCs. In addition, Gel-Ty, which is a gelatin-based enzyme cross-linked hydrogel capable of positioning G-NAC in bone defects, was prepared for effective bone regeneration. The condition of Gel-Ty hydrogel suitable for tissue regeneration was also established by in vitro experiments. The applicability of hASCs encapsulation to hydrogels was confirmed through the biocompatibility test of Gel-Ty hydrogel containing GNPs. Evaluation of bone differentiation and regeneration ability of Ge-Ty/G-NAC hydrogel will be analyzed later through in vitro and in vivo experiments. These results demonstrate that the Gel-Ty/G-NAC complex can be used as an injectable hydrogel for bone regeneration. Our results suggest that it can be widely applied to the bone tissue engineering field.

\section{Acknowledgments}

This research was supported by the Bio \& Medical Technology Development Program of the National Research Foundation (NRF) and funded by the Korean government (MSIP\&MOHW) (No 2017M3A9E4048170).

\section{Disclosure}

The authors report no conflicts of interest in this work.

\section{References}

1. Kinaci A, Neuhaus V, Ring DC. Trends in bone graft use in the United States. Orthopedics. 2014;37(9):e783-e788.

2. Vaccaro AR, Chiba K, Heller JG, et al; North American Spine Society for Contemporary Concepts in Spine Care. Bone grafting alternatives in spinal surgery. Spine J. 2002;2(3):206-215.

3. Clavero J, Lundgren S. Ramus or chin grafts for maxillary sinus inlay and local onlay augmentation: comparison of donor site morbidity and complications. Clin Implant Dent Relat Res. 2003;5(3):154-160. 
4. Marx RE, Morales MJ. Morbidity from bone harvest in major jaw reconstruction: a randomized trial comparing the lateral anterior and posterior approaches to the ilium. J Oral Maxillofac Surg. 1988;46(3):196-203.

5. Bauer TW, Muschler GF. Bone graft materials. An overview of the basic science. Clin Orthop Relat Res. 2000;(371):10-27.

6. Jones JR. Reprint of: Review of bioactive glass: from Hench to hybrids. Acta Biomater. 2015;23(Suppl):S53-S82.

7. García-Gareta E, Coathup MJ, Blunn GW. Osteoinduction of bone grafting materials for bone repair and regeneration. Bone. 2015;81:112-121.

8. Kleinschmidt K, Ploeger F, Nickel J, Glockenmeier J, Kunz P, Richter W. Enhanced reconstruction of long bone architecture by a growth factor mutant combining positive features of GDF-5 and BMP-2. Biomaterials. 2013;34(24):5926-5936.

9. Wang M, Deng Y, Zhou P, et al. In vitro culture and directed osteogenic differentiation of human pluripotent stem cells on peptides-decorated two-dimensional microenvironment. ACS Appl Mater Interfaces. 2015; 7(8):4560-4572

10. Richards DA, Maruani A, Chudasama V. Antibody fragments as nanoparticle targeting ligands: a step in the right direction. Chem Sci. 2017; 8(1):63-77.

11. Tverdokhlebov S, Bolbasov E, Shesterikov E. Scaffold materials based on fluorocarbon composites modified with RF magnetron sputtering. In: Osteogenesis. London, InTech; 2012:83-116.

12. Lee D, Heo DN, Kim HJ, et al. Inhibition of osteoclast differentiation and bone resorption by bisphosphonate-conjugated gold nanoparticles. Sci Rep. 2016;6:27336.

13. Ghosh P, Han G, De M, Kim CK, Rotello VM. Gold nanoparticles in delivery applications. Adv Drug Deliv Rev. 2008;60(11):1307-1315.

14. Shukla R, Bansal V, Chaudhary M, Basu A, Bhonde RR, Sastry M. Biocompatibility of gold nanoparticles and their endocytotic fate inside the cellular compartment: a microscopic overview. Langmuir. 2005; 21(23):10644-10654.

15. Shi X, Wang S, Sun H, Baker JR. Improved biocompatibility of surface functionalized dendrimer-entrapped gold nanoparticles. Soft Matter. 2007;3(1):71-74.

16. Lee D, Heo DN, Lee SJ, et al. Poly(lactide-co-glycolide) nanofibrous scaffolds chemically coated with gold-nanoparticles as osteoinductive agents for osteogenesis. Appl Surf Sci. 2018;432:300-307.

17. Yi C, Liu D, Fong CC, Zhang J, Yang M. Gold nanoparticles promote osteogenic differentiation of mesenchymal stem cells through p38 MAPK pathway. ACS Nano. 2010;4(11):6439-6448.

18. Zhou J, Zhang Y, Li L, Fu H, Yang W, Yan F. Human $\beta$-defensin 3-combined gold nanoparticles for enhancement of osteogenic differentiation of human periodontal ligament cells in inflammatory microenvironments. Int J Nanomedicine. 2018;13:555-567.

19. Heo DN, Ko W-K, Bae MS, et al. Enhanced bone regeneration with a gold nanoparticle-hydrogel complex. J Mater Chem B. 2014;2(11): 1584-1593.

20. Wang N, Qian P, Kumar S, Yan TD, Phan K. The effect of N-acetylcysteine on the incidence of contrast-induced kidney injury: a systematic review and trial sequential analysis. Int J Cardiol. 2016;209:319-327.

21. Mokhtari V, Afsharian P, Shahhoseini M, Kalantar SM, Moini A. A review on various uses of N-acetyl cysteine. Cell J. 2017;19(1):11-17.

22. Yamada M, Tsukimura N, Ikeda T, et al. N-acetyl cysteine as an osteogenesis-enhancing molecule for bone regeneration. Biomaterials. 2013;34(26):6147-6156.

23. Atashi F, Modarressi A, Pepper MS. The role of reactive oxygen species in mesenchymal stem cell adipogenic and osteogenic differentiation: a review. Stem Cells Dev. 2015;24(10):1150-1163.

24. Lee KY, Mooney DJ. Hydrogels for tissue engineering. Chem Rev. 2001;101(7):1869-1880.

25. Park SH, Kwon JS, Lee BS, et al. BMP2-modified injectable hydrogel for osteogenic differentiation of human periodontal ligament stem cells. Sci Rep. 2017;7(1):6603.

26. Mi L, Liu H, Gao Y, Miao H, Ruan J. Injectable nanoparticles/hydrogels composite as sustained release system with stromal cell-derived factor- $1 \alpha$ for calvarial bone regeneration. Int J Biol Macromol. 2017;101: $341-347$.
27. Vishnu Priya M, Sivshanmugam A, Boccaccini AR, et al. Injectable osteogenic and angiogenic nanocomposite hydrogels for irregular bone defects. Biomed Mater. 2016;11(3):035017.

28. Vo TN, Shah SR, Lu S, et al. Injectable dual-gelling cell-laden composite hydrogels for bone tissue engineering. Biomaterials. 2016;83: $1-11$.

29. Koshy ST, Desai RM, Joly P, et al. Click-crosslinked injectable gelatin hydrogels. Adv Healthc Mater. 2016;5(5):541-547.

30. Daniel MC, Astruc D. Gold nanoparticles: Assembly, supramolecular chemistry, quantum-size-related properties, and applications toward biology, catalysis, and nanotechnology. Chem Rev. 2004;104(1):293-346.

31. Yeh YC, Creran B, Rotello VM. Gold nanoparticles: preparation, properties, and applications in bionanotechnology. Nanoscale. 2012;4(6): $1871-1880$

32. Heo DN, Ko WK, Lee HR, et al. Titanium dental implants surfaceimmobilized with gold nanoparticles as osteoinductive agents for rapid osseointegration. J Colloid Interface Sci. 2016;469:129-137.

33. Ko WK, Heo DN, Moon HJ, et al. The effect of gold nanoparticle size on osteogenic differentiation of adipose-derived stem cells. J Colloid Interface Sci. 2015;438:68-76.

34. Zhang XD, Wu D, Shen X, et al. Size-dependent in vivo toxicity of PEGcoated gold nanoparticles. Int J Nanomedicine. 2011;6:2071-2081.

35. Hermanson GT. Bioconjugate Techniques. London: Academic Press; 2013.

36. Pattnaik P. Surface plasmon resonance. Appl Biochem Biotechnol. 2005;126(2):79-92.

37. Jain PK, Qian W, El-Sayed MA. Ultrafast cooling of photoexcited electrons in gold nanoparticle-thiolated DNA conjugates involves the dissociation of the gold-thiol bond. J Am Chem Soc. 2006;128(7): 2426-2433.

38. Zhu ZJ, Ghosh PS, Miranda OR, Vachet RW, Rotello VM. Multiplexed screening of cellular uptake of gold nanoparticles using laser desorption/ ionization mass spectrometry. J Am Chem Soc. 2008;130(43): 14139-14143.

39. Jiang W, Kim BY, Rutka JT, Chan WC. Nanoparticle-mediated cellular response is size-dependent. Nat Nanotechnol. 2008;3(3):145-150.

40. Fan Z, Zhang Y, Fang S, Xu C, Li X. Bienzymatically crosslinked gelatin/hyaluronic acid interpenetrating network hydrogels: preparation and characterization. RSC Adv. 2015;5(3):1929-1936.

41. Kurisawa M, Chung JE, Yang YY, Gao SJ, Uyama H. Injectable biodegradable hydrogels composed of hyaluronic acid-tyramine conjugates for drug delivery and tissue engineering. Chem Commun (Camb). 2005;(34): 4312-4314.

42. Kobayashi S, Uyama H, Kimura S. Enzymatic polymerization. Chem Rev. 2001;101(12):3793-3818.

43. Li Z, Qu T, Ding C, et al. Injectable gelatin derivative hydrogels with sustained vascular endothelial growth factor release for induced angiogenesis. Acta Biomater. 2015;13:88-100.

44. Arora A, Mahajan A, Katti DS. TGF- $\beta 1$ presenting enzymatically crosslinked injectable hydrogels for improved chondrogenesis. Colloids Surf B Biointerfaces. 2017;159:838-848.

45. Bystroňová J, Čigalková I, Wolfová L, Pravda M, Vrana NE, Velebný V. Creating a 3D microenvironment for monocyte cultivation: ECMmimicking hydrogels based on gelatine and hyaluronic acid derivatives. RSC Adv. 2018;8(14):7606-7614.

46. Vial S, Reis RL, Oliveira JM. Recent advances using gold nanoparticles as a promising multimodal tool for tissue engineering and regenerative medicine. Curr Opin Solid State Mater Sci. 2017;21(2):92-112.

47. Lee SJ, Lee HJ, Kim SY, et al. In situ gold nanoparticle growth on polydopamine-coated 3D-printed scaffolds improves osteogenic differentiation for bone tissue engineering applications: in vitro and in vivo studies. Nanoscale. 2018;10(33):15447-15453. 


\section{Supplementary materials}

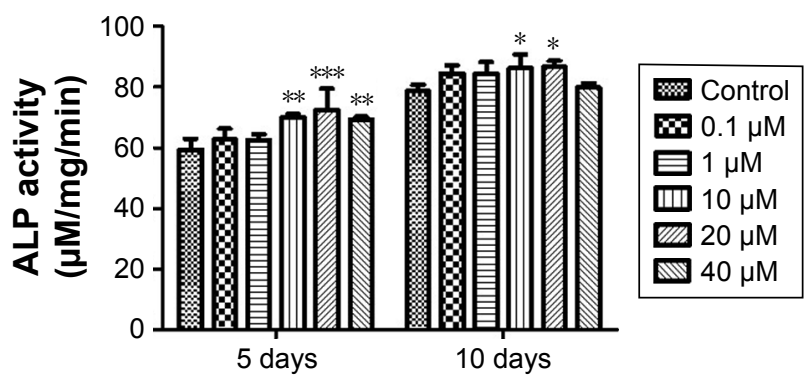

Figure SI Evaluation of ALP activity of hASCs at various concentrations of GNPs below $40 \mu \mathrm{M}$. Results are mean \pm SD of triplicate experiments: $* P<0.05$, $* * P<0.01$, and $* * * P<0.00$ I represent significant difference compared with control group.

Abbreviations: GNPs, gold nanoparticles; hASCs, human-derived stem cells.
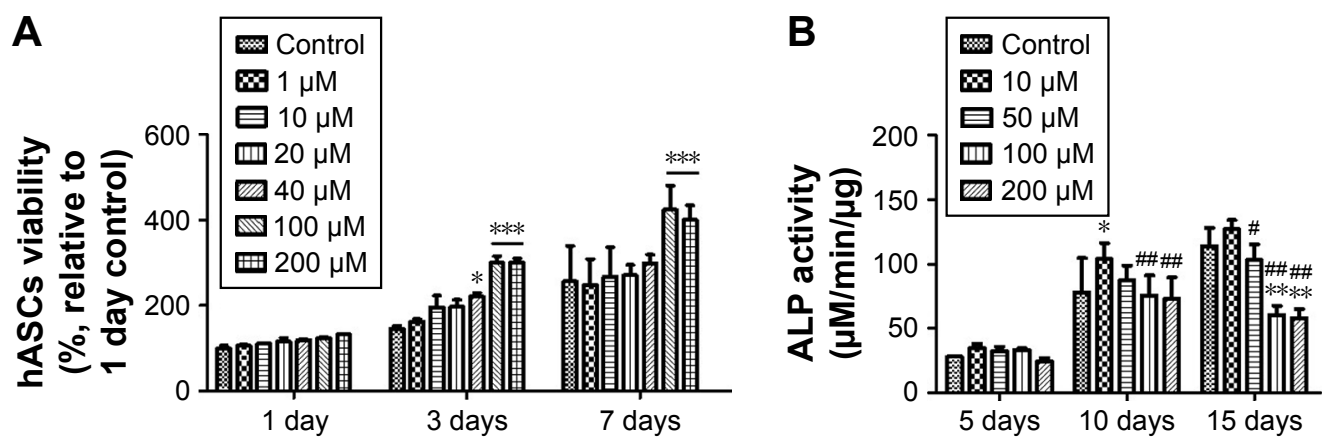

Figure S2 Evaluation of viability $(\mathbf{A})$ and ALP activity $(\mathbf{B})$ of hASCs at various concentrations of G-NAC. Results are mean \pm SD of triplicate experiments: $* P<0.05$, $* * P<0.0$ I, and ${ }^{* * *} P<0.001$ represent significant differences compared with control group, and ${ }^{\#} P<0.05$ and ${ }^{\#} P<0.01$ represent significant differences compared with $10 \mu M$ group. Abbreviations: G-NAC, gold nanoparticles-N-acetyl cysteine; hASCs, human-derived stem cells.
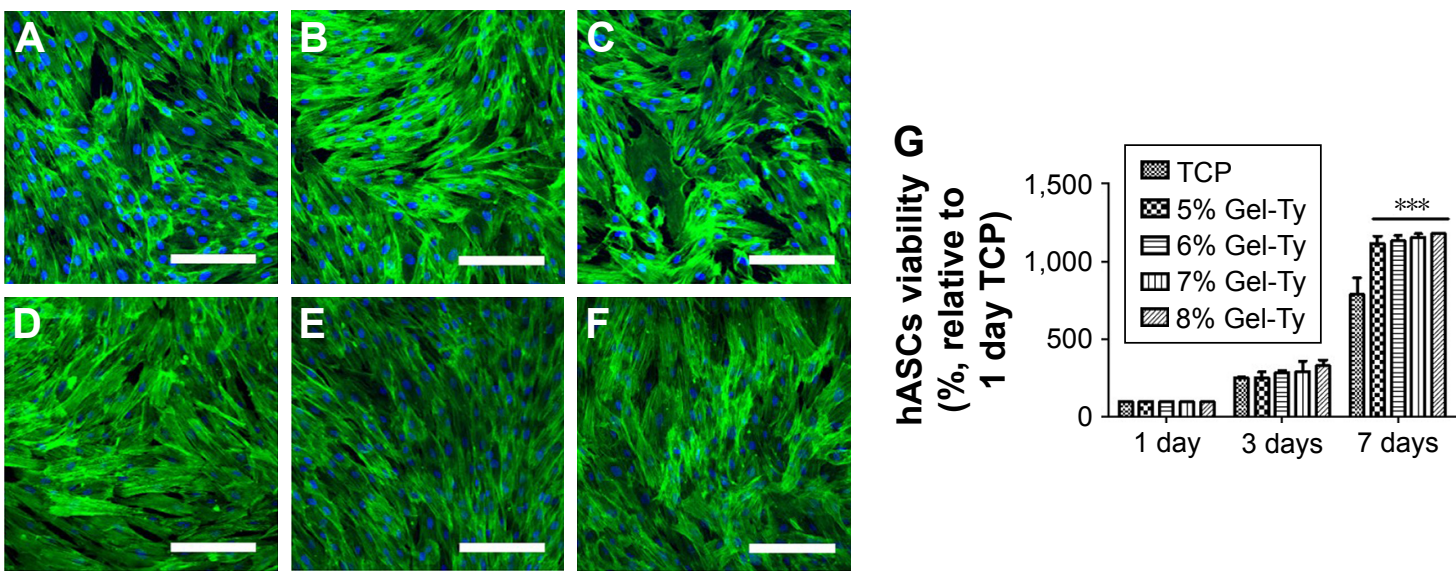

Figure S3 Viability of hASCs on the Gel-Ty hydrogel surface. The CLSM images of 5\% (A, D), 7.5\% (B, E), and I0\% Gel-Ty hydrogel (C, F) after culture during I (A-C) and 3 days (D-F). F-actin of hASCs is green and the nucleus of hASCs is blue. Scale bar is $200 \mu \mathrm{m}$. Images collected at $200 \times$ magnification. The cell viability assay on the Gel-Ty hydrogels for 7 days using EZ-Cytox assay $(\mathbf{G})$. Results are mean \pm SD of triplicate experiments: $* * * P<0.00$ I represent significant differences compared with tissue culture plate group.

Abbreviations: CLSM, confocal laser scanning microscopy; Gel-Ty, gelatin-tyramine; hASCs, human-derived stem cells; TCP, tissue culture plate. 
International Journal of Nanomedicine

Dovepress

\section{Publish your work in this journal}

The International Journal of Nanomedicine is an international, peerreviewed journal focusing on the application of nanotechnology in diagnostics, therapeutics, and drug delivery systems throughout the biomedical field. This journal is indexed on PubMed Central, MedLine, CAS, SciSearch ${ }^{\circledR}$, Current Contents ${ }^{\circledR} /$ Clinical Medicine,
Journal Citation Reports/Science Edition, EMBase, Scopus and the Elsevier Bibliographic databases. The manuscript management system is completely online and includes a very quick and fair peer-review system, which is all easy to use. Visit http://www.dovepress.com/ testimonials.php to read real quotes from published authors.

Submit your manuscript here: http://www.dovepress.com/international-journal-of-nanomedicine-journal 